\title{
Prediction of heme binding residues from protein sequences with integrative sequence profiles
}

\author{
Yi Xiong, Juan Liu*, Wen Zhang, Tao Zeng \\ From IEEE International Conference on Bioinformatics and Biomedicine 2011 \\ Atlanta, GA, USA. 12-15 November 2011
}

\begin{abstract}
Background: The heme-protein interactions are essential for various biological processes such as electron transfer, catalysis, signal transduction and the control of gene expression. The knowledge of heme binding residues can provide crucial clues to understand these activities and aid in functional annotation, however, insufficient work has been done on the research of heme binding residues from protein sequence information.

Methods: We propose a sequence-based approach for accurate prediction of heme binding residues by a novel integrative sequence profile coupling position specific scoring matrices with heme specific physicochemical properties. In order to select the informative physicochemical properties, we design an intuitive feature selection scheme by combining a greedy strategy with correlation analysis.

Results: Our integrative sequence profile approach for prediction of heme binding residues outperforms the conventional methods using amino acid and evolutionary information on the 5 -fold cross validation and the independent tests.
\end{abstract}

Conclusions: The novel feature of an integrative sequence profile achieves good performance using a reduced set of feature vector elements.

\section{Background}

The heme-protein interactions are involved in a wide range of biological processes such as electron transfer, catalysis, signal transduction and control of gene expression [1]. To better understand the mechanism of hemeprotein interactions and aid in heme related functional annotation, it is crucial to characterize and identify the binding sites of heme proteins[2]. It is well known that experimental techniques towards the determination of heme binding sites are prohibitively time-consuming and labour-intensive. Therefore, computational approaches are significantly in need for a rapid, highthroughput prediction of heme binding residues.

Recently, a pioneering method HemeBIND [3] is specifically designed to predict heme binding residues on heme proteins. At present, HemeBIND provides two complementary methods to distinguish heme binding

\footnotetext{
* Correspondence: liujuan@whu.edu.cn

School of Computer, Wuhan University, Wuhan 430072, China
}

sites from the rest of heme proteins. The main method integrates both sequence and structural features including evolutionary profile, solvent accessibility, depth, and protrusion index. Although the structural information can provide helpful insights for characterizing heme binding residues, there are currently only small fractions of 3D structures available for the heme proteins, which will limit the application scope of the structure-based methods. Therefore, HemeBIND also provides an alternative sequence-based method which can predict heme binding sites when only sequence information is available for heme proteins. The sequence-based classifier is constructed by the evolutionary information of amino acid sequences in the form of position specific scoring matrices (PSSM) that is generated by multiple sequence alignments.

In fact, in addition to PSSM, the physicochemical properties with high interpretability are also commonly used in the prediction of protein function from sequences[4-8]. Our previous work [9] also confirms the 
role of physicochemical properties in DNA-binding residues. However, to the best of our knowledge, no related work has incorporated physicochemical and biological properties from the Amino Acid Index (AAindex) database $[10,11]$ to analyze and predict heme binding residues.

In the present study, we focus on the prediction of sequence-based heme binding sites, and attempt to integrate the physicochemical properties into PSSM, to provide additional insights to the heme binding residues and advance the prediction performance in actual applications. First, we use an intuitive feature selection scheme to choose an informative and compact subset of physicochemical descriptors in AAindex database. Then, we propose a novel integrative sequence profile, which is generated by coupling PSSM with the selected physicochemical properties. Evaluation experiments by using 5 -fold cross validation on the training set and on the independent test demonstrate that our proposed approach outperforms the conventional methods based on PSSM profiles for prediction of heme binding residues.

\section{Methods}

\section{Datasets}

For training and testing, we used the datasets of heme proteins in previous studies $[3,12]$. The training set consists of 75 heme protein chains, derived from the nonredundant dataset of 89 heme proteins prepared by Fufezan et al [12]. After removing 14 chains whose HET group codes are not labelled as "HEM", we obtained the remaining 75 heme protein chains as the training set (PHeme-75). Since the heme proteins in PHeme-75 were constructed before March 2007, we used the other heme proteins collected after March 2007 as the testing set (PHeme-72) [3]. PHeme-72 is a nonredundant set of 72 heme protein chains, sharing no more than $30 \%$ sequence identity with any one of the 75 chains in the training set.

Following previous studies[3,13-16], we used the Ligand Protein Contact server to assign heme binding and nonbinding residues for the protein chains in the datasets. Among the training set of PHeme-75, we obtained 18584 residues with atomic coordinates, of which about $13.5 \%$ are heme binding sites. On the testing set of Pheme-72, there are 18581 residues, of which about $14.3 \%$ are heme binding residues.

\section{Feature construction}

To build a classifier that can identify heme-binding residues from protein sequences, we constructed an effective strategy for integrating various features based on evolutionary profiles and physicochemical properties. For each target (or central) residue, the feature vector was constructed by the sliding window on a consecutive sequence for including the environmental information. In our study, we set $w=17$ as the optimal size for building the sliding window (see details in Results section).

\section{Evolutionary information}

This was obtained as the PSSMs generated by three iterations of PSI-BLAST [17] searches against NCBI nonredundant database (ftp://ftp.ncbi.nlm.nih.gov/blast/ $\mathrm{db} /$ ). The log odds values of 20 amino acid substitutions at a given alignment position were utilized to represent the evolutionary profile of a residue. The PSSM values were scaled to the range $0[1]$ by a standard logistic function [18]. One additional bit is utilized to deal with the terminal spanning windows. For the window size $w$, a vector of size $(20+1)^{*} w$ is used for representing a sample.

\section{Physicochemical property}

Physicochemical properties (PP) are the most intuitive features for biochemical reactions and are widely applied in bioinformatics studies [4]. AAindex (http://www.genome.ad.jp/aaindex/) database is the collection of numerical indices representing various physicochemical properties of amino acids. The AAindex 1 section of the AAindex database currently contains 544 amino acid indices. We removed the indices with missing values in AAindex1, with 531 entries left for use in our study. The raw values of these physicochemical properties were normalized to zero mean and unit standard deviation according to:

$$
\begin{aligned}
& P_{i j}^{\prime}=\frac{P_{i j}-\mu}{\sigma} \\
& \mu=\frac{1}{20} \sum_{j=1}^{20} P_{i j} \\
& \sigma=\sqrt{\frac{1}{20} \sum_{j=1}^{20}\left(P_{i j}-\mu\right)^{2}}
\end{aligned}
$$

where $P_{i j}$ is the raw value of the $i$-th physicochemical property for the $j$-th amino acid type.

In order to select a subset of informative physicochemical properties, we first measured and ranked the predictive power of these 531 individual indices for correctly classifying all residues with atomic coordinates in Pheme-75 dataset using the area under the receiver operating characteristic curve $(A U C)$. At this stage, no classifier is built so that no cross-validation scheme is required to calculate the $A U C$ scores [19]. 
The $A U C$ for an amino acid type is calculated in the same way as it would be for a classifier output. Each sample is associated with a feature value (e.g. an amino acid scale for a physicochemical property) and a positive or negative class label. A sample set is therefore converted into a sequence of feature values with an associated positive or negative label. The receiver operating characteristic curve and its $A U C$ is calculated over this sequence.

We then designed a greedy approach in combination with correlation analysis for feature selection by constructing and assessing a series of heme binding sites predictors using 5-fold cross validation on the Pheme75 dataset. Figure 1 shows the workflow of the iterative feature selection process. In this implementation, let $C$ be the set of candidate features to be selected, and $S$ be the set of features already selected. Initially, $C$ was composed of the preselected features via $A U C$ scores and $S$ was empty. The features from $C$ were then iteratively selected into $S$ until $C$ was empty. At the end, the features in $S$ were used as the final feature subset in the whole feature selection process.

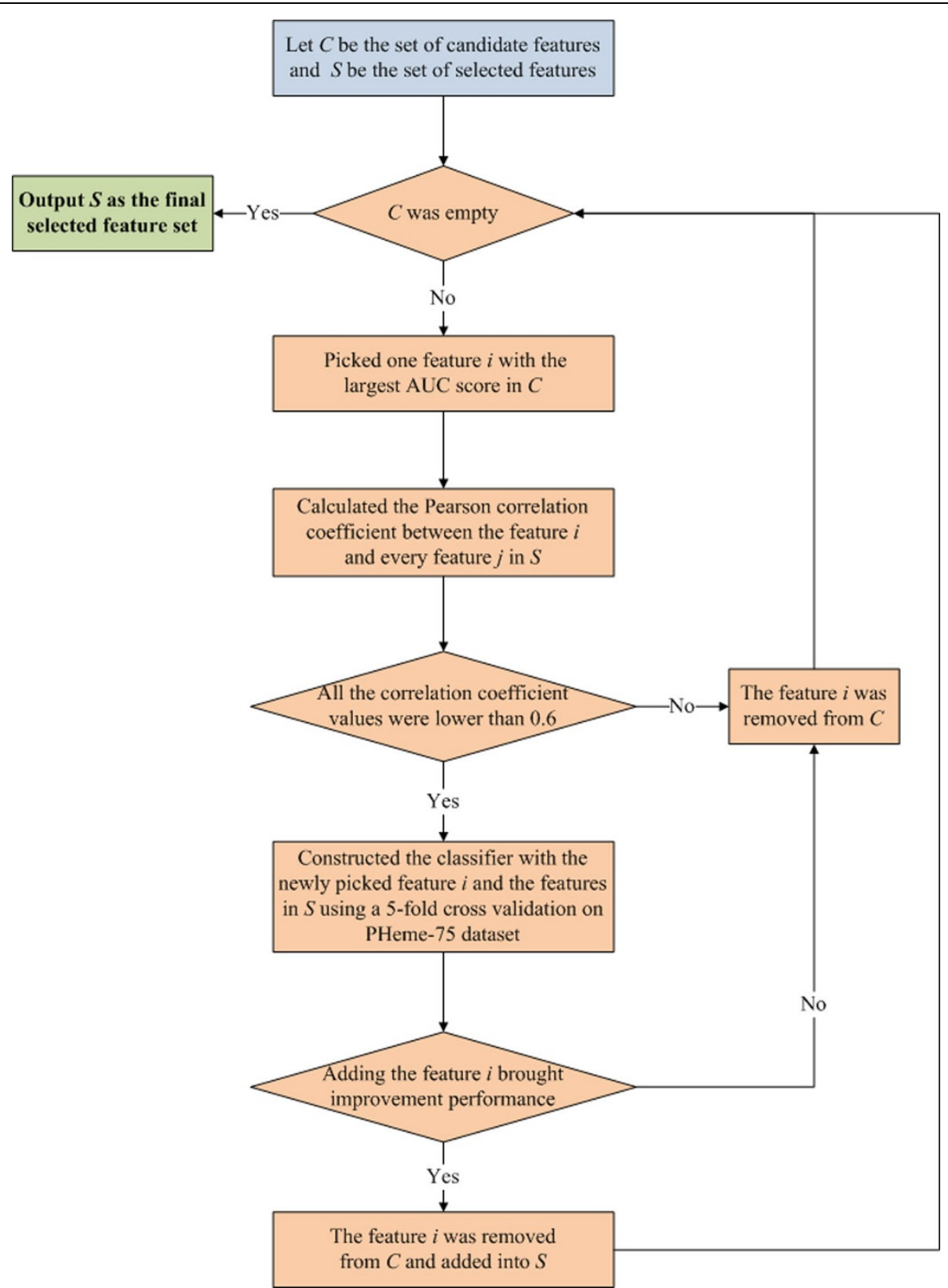

Figure 1 Workflow of the proposed iterative feature selection process. 


\section{Integrative sequence profile}

For the purpose of combining the predictive power of PSSM and PP, it is conventional to concatenate the vector elements of the PSSM and PP into a longer feature vector. Instead of using a concatenated vector with a high dimension, we implemented a condensed integrative profile by coupling PSSM with PP (called PSSMPP). The feature set of PSSMPP was constructed by summing up the 20 amino acid columns of the PSSM, weighted by the corresponding 20 amino acid values for a certain physicochemical property. Figure 2 presents an example for generating the PSSMPP profile vector. In the PSSMPP, the entry $F_{i p}$ of row $i$ in the PSSM for a considering physicochemical property $p$ is defined as follows, in much the similar way as previous work [20-22].

$$
F_{i p}=\sum_{j=1}^{20} w_{p j} \cdot M_{i j}
$$

where

(1) $i$ is the index of a position in the protein sequence;

(2) $w_{p j}$ is the normalized value of physicochemical property $p$ for the $j$-th amino acid;

(3) $M_{i j}$ is the scaled value of the $j$-th type of amino acid in the position $i$ of the PSSM.

\section{Model construction and evaluation}

Support Vector Machines (SVMs) were applied to prediction of heme binding sites in our experiments. The SVMs are based on a rigorous statistical learning theory and have high generalization ability [23]. The SVM algorithms have demonstrated powerful performance in similar bioinformatics studies [24-27]. In this study, the SVM models were implemented with the radial basis function as a kernel using the e1071 library in R (http:// cran.r-project.org/web/packages/e1071/), which provides the interface to the LibSVM. The models were first evaluated by 5 -fold cross validation on the training set. Each classifier was trained using a data set comprising all positive samples from the cross validation fold and an equal number of randomly chosen negative samples. The models were further evaluated by the independent test on PHeme-72.

The performance of classification algorithms can be assessed by these metrics: accuracy $(A C C)$, sensitivity $(S N$, also called recall), specificity $(S P)$, precision $(P R)$, Matthew's correlation coefficient $(M C C)$ and F-measure $\left(F_{1}\right)$. These metrics are calculated using the numbers of true positives $(T P)$, false positives $(F P)$, true negatives $(T N)$ and false negatives $(F N)$ for each classifier. The performance measures are defined by the following equations.

$$
\begin{aligned}
& A C C=\frac{T P+T N}{T P+T N+F P+F N} \\
& S N=\frac{T P}{T P+F N} \\
& S P=\frac{T N}{T N+F P} \\
& P R=\frac{T P}{T P+F P} \\
& M C C=\frac{T P \times T N-F P \times F N}{\sqrt{(T P+F N) \times(T P+F P) \times(T N+F P) \times(T N+F N)}} \\
& \mathrm{F}_{1}=\frac{2 \times S N \times P R}{S N+P R}
\end{aligned}
$$

The receiver operating characteristic curve is a plot of the sensitivity versus (1-specificity) for a binary classifier at varying thresholds. The $A U C$ was used as a main measure of classification performance throughout our work.

\section{Results}

Analysis of the selected physicochemical properties

Although some of the individual amino acid indices show modest discrimination abilities for distinguishing binding from nonbinding residues, the inter-feature redundancy makes it fail to improve the classification performance when they are combined together (data not shown). To rectify this problem, we performed a greedy feature selection approach in combination with correlation analysis to reach an optimal subset of features. Following the proposed iterative feature selection process, we obtained a subset of four physicochemical properties, which are listed in Table 1.

From Table 2, most of the correlation coefficients among them were sufficiently low, which can partly justify using them in combination. These derived 4 physicochemical properties are related to alpha propensity [28], beta propensity[29] and the preference for linker regions of amino acids[30]. For example, when we use the amino acid scale SUYM030101 for analysis of heme binding and nonbinding residues, the heme binding residues are more abundant in the types of amino acids with high propensity in linker regions.

\section{Prediction performance on Pheme-75 dataset using various feature sets}

In this section, we evaluated the classification performance of different feature sets using 5-fold cross 


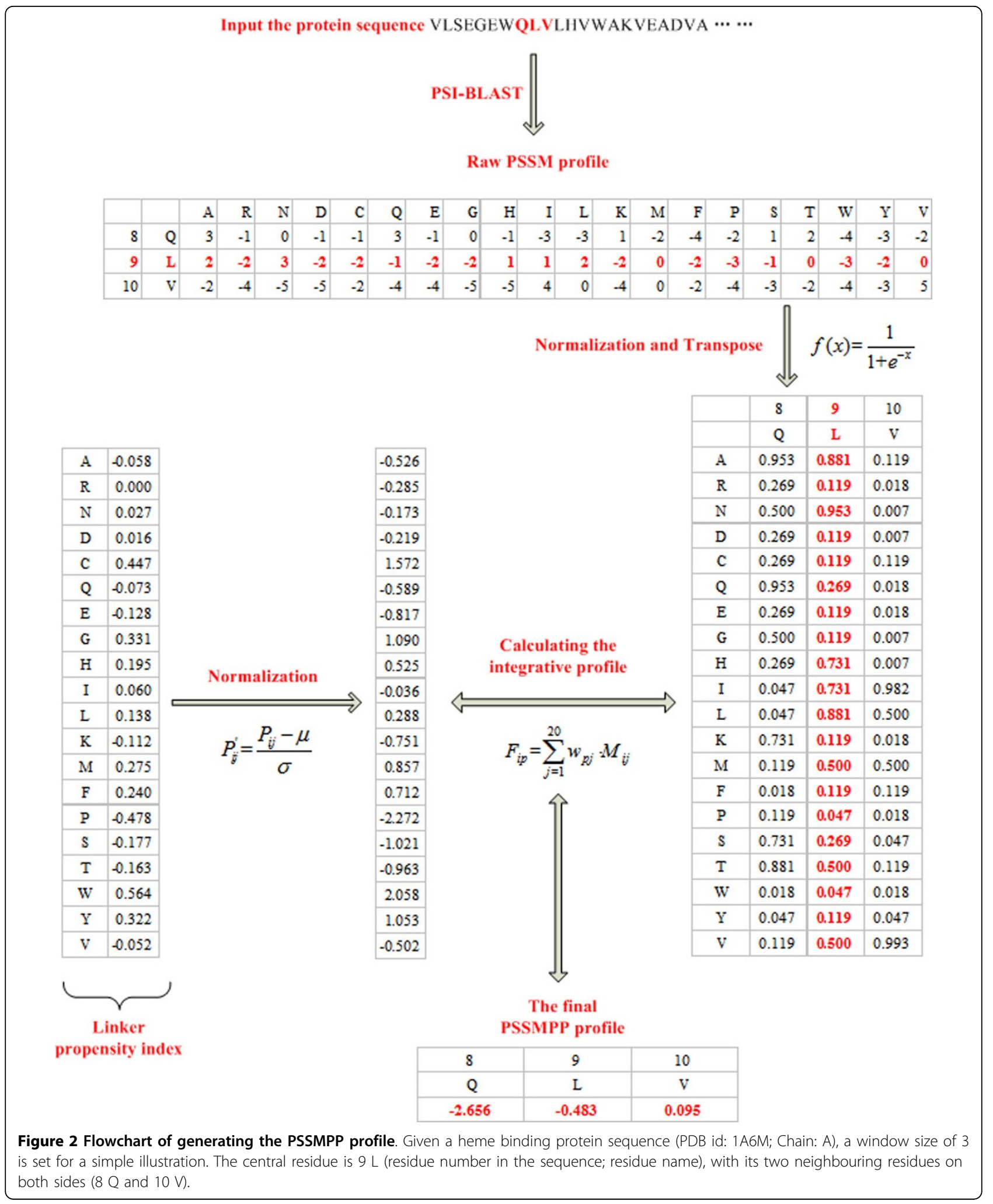

validation on Pheme-75. Since the sliding window strategy was used to include the environment information, we should try different window sizes in order to find out an optimal window length. Figure 3 shows the performance of various features at varying window size from 1 to 29 . In this study we adopted the window size 
Table 1 The list of the selected subset of physicochemical properties on Pheme-75 dataset

\begin{tabular}{lll}
\hline ID & Description & AUC \\
\hline QIAN880117 & Weights for beta-sheet at the window position of -3 & 0.598 \\
AURR980103 & Normalized positional residue frequency at helix termini N" & 0.593 \\
AURR980118 & Normalized positional residue frequency at helix termini C" & 0.583 \\
SUYM030101 & Linker propensity index & 0.573 \\
\hline
\end{tabular}

of 17 unless otherwise stated, since all features achieved highest $A U C$ values at this size. A closer examination of Figure 3 reveals that the conventional representation method of concatenating the vector elements of the PSSM and PP (PSSM +PP) yields marginally higher performance than PSSM at the cost of training time. However, the integrative profile of PSSMPP consistently outperforms all other feature sets when the window size is larger than 9. When using the optimal window size of 17 , it is more obviously shown that PSSMPP performs better than the PSSM and the concatenated combination of PSSM with PP (PSSM +PP).

Table 3 presents the detailed metrics of the SVM classifiers using various features at the window size of 17 . It is worth mentioning that the PSSMPP feature set improves precision and other metrics at the expense of the recall, but without dramatically compromising the recall measure. The moderate improvement of the overall performance is promising, considering the fact that PSSMPP used a significantly lower size of $85((4+1)$ " 17$)$ dimensions in the input vectors than the sizes of 357 and $425((20+4+1) * 17)$, for PSSM and (PSSM+PP), respectively. The result is in agreement with the finding of the previous work [31] that a simple representation of the feature space could be much more powerful and efficient than the original data with all information included.

\section{Independent test on PHeme-72 dataset and comparison with HemeBIND}

A true test of any prediction approach is to make predictions for the unseen dataset not utilized in training. In the section, we evaluated the prediction performance on an independent set of PHeme-72 using the best model trained on Pheme-75. As shown in Table 4, the testing performance of our model is not worse but even better than the training performance when using the same feature sets. The result suggests that the features we used here are not overfitting to the training set. In fact, for fairly comparing the predictive power of different features, we trained the SVM models using default parameters, resulting in the fact that the performance of the independent test set is even higher than that of the training set.

In HemeBIND [3], two sequence-based classifiers were built by using amino acid binary pattern and PSSM, respectively. The authors have shown that the classifier based on PSSM significantly improved the prediction performance of the method based on unique representations (or binary encoding) of amino acid sequence and its environment. We implemented these two models, and compared our PSSMPP method with them using the same training and testing set, and the same definition of heme binding residues. Table 4 shows that our PSSMPP approach performs better than the binary and PSSM methods. Both in our work and Liu and Hu's study[3], the PSSM method outperforms the binary method.

\section{Conclusions}

The main goal of the current study is to provide valuable insights to the heme binding residues and improve the classification performance based on heme protein sequences. In order to mine the informative physicochemical descriptors for heme binding residues, we designed a greedy approach in combination with correlation analysis for feature selection. Based on the selected physicochemical properties, we implemented an integrative sequence profile by coupling PSSM with four heme related physicochemical properties. The novel feature of PSSMPP achieves good performance using a reduced set of feature vector elements, whose size is significantly smaller than that of the conventional feature sets (i.e., PSSM). We believed that the reduced set of an

Table 2 The correlation coefficients among the four physicochemical properties on Pheme-75 dataset

\begin{tabular}{lllll}
\hline & QIAN880117 & AURR980103 & AURR980118 & SUYM030101 \\
\hline QIAN880117 & - & - & - & - \\
AURR980103 & 0.020 & - & - & - \\
AURR980118 & -0.053 & 0.557 & - & - \\
SUYM030101 & 0.107 & 0.104 & 0.286 & - \\
\hline
\end{tabular}




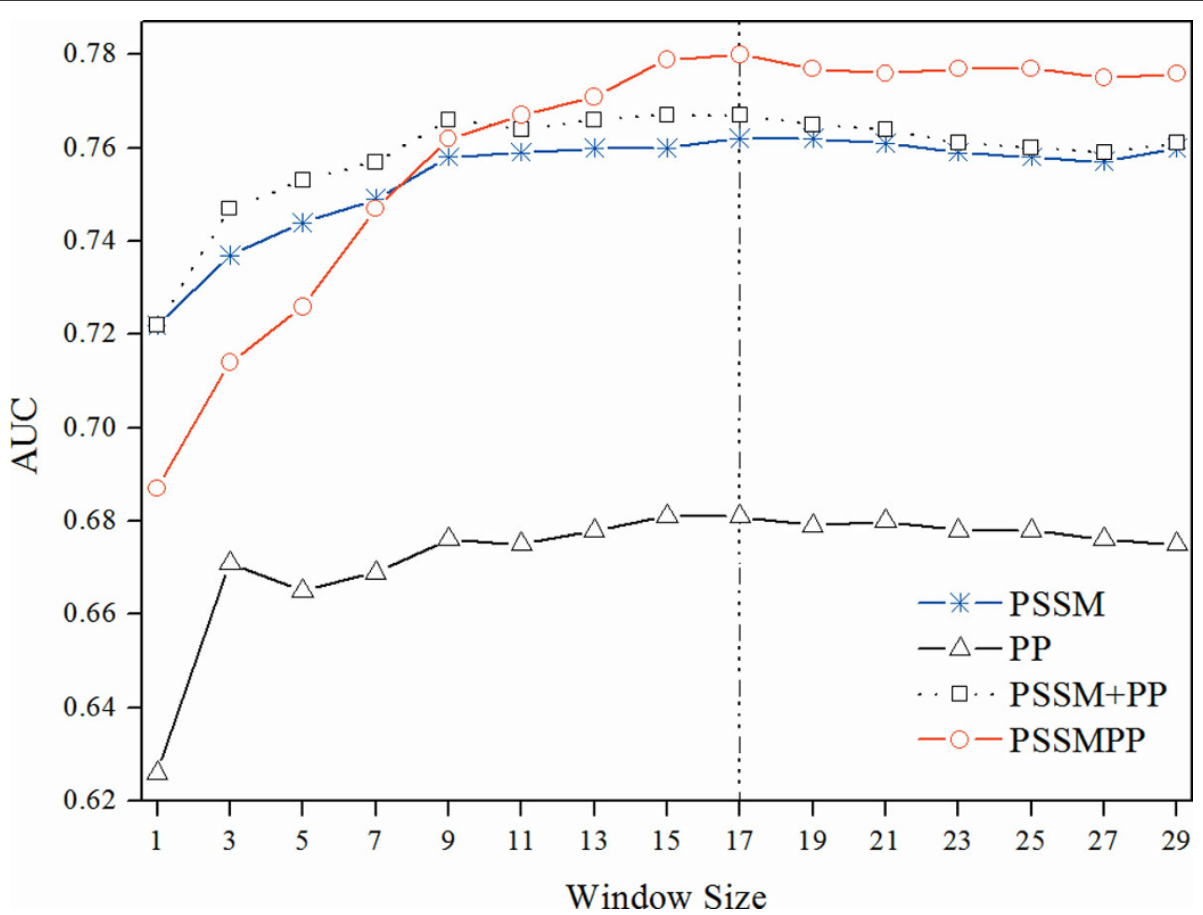

Figure 3 Performance comparison of different features using 5-fold cross validation on Pheme-75 dataset at varying window sizes

Table 3 Performance of different features on PHeme-75 dataset using 5-fold cross validation

\begin{tabular}{llllllll}
\hline Feature & ACC(\%) & SN (\%) & SP (\%) & PR(\%) & MCC & F $_{\mathbf{1}}$ & AUC \\
\hline PSSM & 66.3 & $\mathbf{7 3 . 4}$ & 65.3 & 25.4 & 0.272 & 0.374 & 0.762 \\
PP & 62.9 & 63.4 & 62.7 & 21.4 & 0.184 & 0.319 & 0.681 \\
PSSM+PP & 67.8 & 72.1 & 67.2 & 26.2 & 0.279 & 0.381 & 0.767 \\
PSSMPP & $\mathbf{7 1 . 1}$ & 71.0 & $\mathbf{7 1 . 1}$ & $\mathbf{2 8 . 5}$ & $\mathbf{0 . 3 0 6}$ & $\mathbf{0 . 4 0 3}$ & $\mathbf{0 . 7 8 0}$
\end{tabular}

Table 4 Performance comparison of different methods on the independent test set of PHeme-72

\begin{tabular}{llllllll}
\hline Feature & $\mathbf{A C C}(\%)$ & SN (\%) & SP (\%) & PR(\%) & MCC & $\mathbf{F}_{\mathbf{1}}$ & AUC \\
\hline Binary & 67.9 & 61.8 & 68.9 & 24.9 & 0.225 & 0.355 & 0.718 \\
PSSM & 65.9 & $\mathbf{7 5 . 2}$ & 64.3 & 26.0 & 0.280 & 0.386 & 0.768 \\
PSSMPP & $\mathbf{7 1 . 7}$ & 71.6 & $\mathbf{7 1 . 8}$ & $\mathbf{2 9 . 7}$ & $\mathbf{0 . 3 1 9}$ & $\mathbf{0 . 4 2 0}$ & $\mathbf{0 . 7 9 0}$ \\
\hline
\end{tabular}

integrative sequence profile feature can potentially be expanded to predict other functional residues on proteins.

List of abbreviations used

PSSM: Position Specific Scoring Matrices; AAindex: Amino Acid Index; PP: Physicochemical Property; AUC: Area Under Curve; SVM: Support Vector Machine; ACC: Accuracy; SN: Sensitivity; SP: Specificity; PR: Precision; MCC: Matthew's Correlation Coefficient; Fl: F-measure; TP: True Positive; FP: False Positive; TN: True Negative; FN: False Negative.

\section{Acknowledgements}

This work is supported by the grants from the National Science Foundation of China (60970063, 61103126), the program for New Century Excellent Talents in Universities (NCET-10-0644), the Ph.D. Programs Foundation of Ministry of Education of China (20090141110026, 20100141120049), the Fundamental Research Funds for the Central Universities (6081007, 3101054), and the Natural Science Foundation of Hubei Province (No. 2011CDB454).

This article has been published as part of Proteome Science Volume 10 Supplement 1, 2012: Selected articles from the IEEE International Conference on Bioinformatics and Biomedicine 2011: Proteome Science. The full contents of the supplement are available online at http://www.proteomesci. com/supplements/10/S1

\section{Authors' contributions}

$Y X$ and $J L$ conceived and designed the experiments. $Y X$ implemented the prediction method. YX, WZ and TZ analyzed the data and wrote the manuscript. $J$ finalized the manuscript. All authors read and approved the final manuscript.

\section{Competing interests}

The authors declare that they have no competing interests.

Published: 21 June 2012

\section{References}

1. Schneider S, Marles-Wright J, Sharp KH, Paoli M: Diversity and conservation of interactions for binding heme in b-type heme proteins. Nat Prod Rep 2007, 24:621-630.

2. Smith $L$, Kahraman A, Thornton JM: Heme proteins-diversity in structural characteristics, function, and folding. Proteins 2010, 78:2349-2368.

3. Liu R, Hu J: HemeBIND: a novel method for heme binding residue prediction by combining structural and sequence information. $B M C$ Bioinformatics 2011, 12:207.

4. Tung CW, Ho SY: Computational identification of ubiquitylation sites from protein sequences. BMC Bioinformatics 2008, 9:310. 
5. Tung CW, Ho SY: POPI: predicting immunogenicity of MHC class I binding peptides by mining informative physicochemical properties. Bioinformatics 2007, 23:942-949.

6. Huang $H L$, Lin IC, Liou YF, Tsai CT, Hsu KT, Huang WL, Ho SJ, Ho SY: Predicting and analyzing DNA-binding domains using a systematic approach to identifying a set of informative physicochemical and biochemical properties. BMC Bioinformatics 2011, 12(Suppl 1):S47.

7. Xia JF, Zhao XM, Huang DS: Predicting protein-protein interactions from protein sequences using meta predictor. Amino Acids 2010, 39:1595-1599.

8. Xia JF, Wang SL, Lei YK: Computational methods for the prediction of protein-protein interactions. Protein Pept Lett 2010, 17:1069-1078.

9. Xiong Y, Liu J, Wei DQ: An accurate feature-based method for identifying DNA-binding residues on protein surfaces. Proteins 2011, 79:509-517.

10. Kawashima S, Pokarowski P, Pokarowska M, Kolinski A, Katayama T, Kanehisa M: AAindex: amino acid index database, progress report 2008. Nucleic Acids Res 2008, 36:D202-205.

11. Kawashima S, Kanehisa M: AAindex: amino acid index database. Nucleic Acids Res 2000, 28:374.

12. Fufezan C, Zhang J, Gunner MR: Ligand preference and orientation in band c-type heme-binding proteins. Proteins 2008, 73:690-704.

13. Mishra NK, Raghava GP: Prediction of FAD interacting residues in a protein from its primary sequence using evolutionary information. $B M C$ Bioinformatics 2010, 11(Suppl 1):S48.

14. Chauhan JS, Mishra NK, Raghava GP: Prediction of GTP interacting residues, dipeptides and tripeptides in a protein from its evolutionary information. BMC Bioinformatics 2010, 11:301.

15. Ansari HR, Raghava GP: Identification of NAD interacting residues in proteins. BMC Bioinformatics 2010, 11:160.

16. Chauhan JS, Mishra NK, Raghava GP: Identification of ATP binding residues of a protein from its primary sequence. BMC Bioinformatics 2009, 10:434.

17. Altschul SF, Madden TL, Schaffer AA, Zhang JH, Zhang Z, Miller W, Lipman DJ: Gapped BLAST and PSI-BLAST: a new generation of protein database search programs. Nucleic Acids Res 1997, 25:3389-3402.

18. Jones DT: Protein secondary structure prediction based on positionspecific scoring matrices. J Mol Biol 1999, 292:195-202.

19. Maetschke SR, Yuan Z: Exploiting structural and topological information to improve prediction of RNA-protein binding sites. BMC Bioinformatics 2009, 10:341.

20. Ma X, Guo J, Wu J, Liu H, Yu J, Xie J, Sun X: Prediction of RNA-binding residues in proteins from primary sequence using an enriched random forest model with a novel hybrid feature. Proteins 2011, 79:1230-1239.

21. Shimizu K, Hirose S, Noguchi T: POODLE-S: web application for predicting protein disorder by using physicochemical features and reduced amino acid set of a position-specific scoring matrix. Bioinformatics 2007, 23:2337-2338.

22. Su CT, Chen CY, Ou YY: Protein disorder prediction by condensed PSSM considering propensity for order or disorder. BMC Bioinformatics 2006, 7:319.

23. Cortes C, Vapnik V: Support-vector networks. Machine learning 1995, 20:273-297.

24. Xia JF, Zhao XM, Song J, Huang DS: APIS: accurate prediction of hot spots in protein interfaces by combining protrusion index with solvent accessibility. BMC Bioinformatics 2010, 11:174

25. Liu R, Jiang $W$, Zhou Y: Identifying protein-protein interaction sites in transient complexes with temperature factor, sequence profile and accessible surface area. Amino Acids 2010, 38:263-270.

26. Xiong Y, Xia J, Zhang W, Liu J: Exploiting a Reduced Set of Weighted Average Features to Improve Prediction of DNA-Binding Residues from 3D Structures. PLoS One 2011, 6:e28440.

27. Chen K, Mizianty MJ, Kurgan L: ATPsite: sequence-based prediction of ATP-binding residues. Proteome Sci 2011, 9(Suppl 1):S4.

28. Aurora R, Rose GD: Helix capping. Protein Science 1998, 7:21-38

29. Qian N, Sejnowski TJ: Predicting the secondary structure of globular proteins using neural network models. J Mol Biol 1988, 202:865-884

30. Suyama M, Ohara O: DomCut: prediction of inter-domain linker regions in amino acid sequences. Bioinformatics 2003, 19:673-674.

31. Chen P, Li J: Sequence-based identification of interface residues by an integrative profile combining hydrophobic and evolutionary information. BMC Bioinformatics 2010, 11:402.
doi:10.1186/1477-5956-10-S1-S20

Cite this article as: Xiong et al:: Prediction of heme binding residues from protein sequences with integrative sequence profiles. Proteome Science 2012 10(Suppl 1):S20.

\section{Submit your next manuscript to BioMed Central and take full advantage of:}

- Convenient online submission

- Thorough peer review

- No space constraints or color figure charges

- Immediate publication on acceptance

- Inclusion in PubMed, CAS, Scopus and Google Scholar

- Research which is freely available for redistribution

Submit your manuscript at www.biomedcentral.com/submit
Ciomed Central 